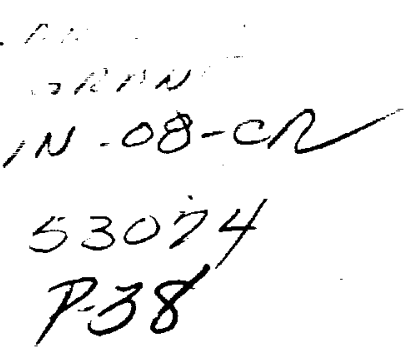

\title{
Rapid Near-Optimal Aerospace Plane Trajectory Generation and Guidance
}

\author{
Final Report
}

November, 1991

Reporting Period: 12/1/88 - 8/31/91

Research Supported by the NASA Langley Research Center

NASA Grant Number: NAG-1-922

Principal Investigators: A. J. Calise

Research Assistants: J. E. Corban and N. Markopoulos

NASA Contract Monitor: D. D. Moerder

School of Aerospace Engineering

Georgia Institute of Technology

Atlanta, Georgia $\mathbf{3 0 3 3 2}$ 


\section{Table of Contents}

Page

I. Summary from previous reporting periods ...................................... 1

II. Progress this reporting period ........................................................ 4

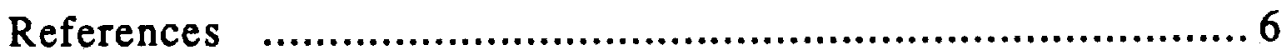

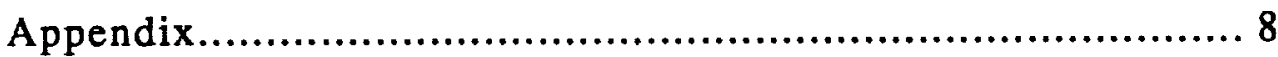

\section{List of Figures}

Figure

1. Energy climb paths for an F-8 aircraft. 29

2. Evaluation of $\varepsilon(E)$ for an F-8 aircraft. 30

3. Energy climb paths for an F-15 aircraft. 31

4. Evaluation of $\varepsilon(E)$ for an F-15 aircraft. 32

5. Energy climb paths for a short haul transport aircraft. 33

6. Evaluation of $\varepsilon(E)$ for a short haul transport aircraft. 34

7. Energy climb paths for a generic hypersonic vehicle 35

8. Evaluation of $\varepsilon(E)$ for a generic hypersonic vehicle 36

\section{List of Tables}

Table

Page

1 Estimation of $\varepsilon_{\mathrm{UB}}$ based on Eq. (32)

17 


\section{Summary from previous reporting periods}

This is the final progress report covering the complete period from December 1,1988 to August 31, 1991, funded under the NASA Contract NAG-1-922. The research effort was directed toward the problems of real-time trajectory optimization and guidance law development for National Aerospace Plane applications. In particular, singular perturbation methods were used to develop guidance algorithms suitable for on board, real-time implementation.

The bulk of the work completed during the period is summarized in three previous reports, listed as references [1]-[3]. Reference [4] cites the final progress report from the previous project (funded under the NASA Contract NAG-1-784).

The work completed during the period from December 1, 1988 to June 30, 1989 (See Ref. [1]) consisted primarily of extensions to the analysis reported in reference [4] to include a number of important considerations. In particular, the vehicle model was extended to include angle of attack effects, the thrust vector component normal to the velocity vector, and flight in the subsonic and supersonic regimes. A multi-mode propulsion system consisting of turbojet, ramjet, scramjet and rocket engines was assumed and simple models for thrust generation and fuel consumption were adopted for each engine cycle. The state-space was further constrained by considering a maximum allowable heating rate. Singular perturbation methods were applied to this more realistic model, leading to a simple algorithm suitable for generating a nearly-fuel-optimal altitude profile in real time. A simple iterative algorithm was derived that approximates the optimal engine transition points and the regions of cycle overlap. Feedback linearization was employed to derive an angle of attack controller which can be used to guide the vehicle along the nearlyfuel-optimal altitude profile in simulations of flight within the atmosphere. A computer subroutine based on the space shuttle explicit guidance algorithm was written to handle the exoatmospheric phase of ascent guidance which allows for the simulation of insertion into orbit. The resulting software was employed to examine the influence of the added model complexity on the fuel-optimal ascent trajectories and the performance of the guidance algorithms.

During the second reporting period, up to December 31, 1989 (See Ref. [2]) general problems associated with on-board trajectory optimization, propulsion system cycle selection, and with the synthesis of guidance laws were addressed for ascent to low-Earthorbit of an air-breathing, single-stage-to-orbit vehicle. The work built directly upon the analytical results of reference [1]. A good portion of the work focused on making improvements to the vehicle models employed. The NASA "Generic Hypersonic Aerodynamic Model Example" and the "Langley Accelerator" aerodynamic data sets were acquired and implemented. Work pertaining to the development of purely analytic aerodynamic models also continued at a low level. A generic model of a multi-mode propulsion system was developed that includes turbojet, ramjet, scramjet, and rocket engine cycles. Provisions were made in the dynamic model for a component of thrust normal to the flight path. Computational results, which characterize the nonlinear sensitivity of scramjet performance to changes in vehicle angle of attack, were obtained and incorporated into the engine model. Additional trajectory constraints were also introduced. The constraints treated were: maximum dynamic pressure, maximum aerodynamic heating rate per unit area, angle of attack and lift limits, and limits on acceleration both along and normal to the flight path.

The remainder of the research effort during the second period focused, for the most part, on required modifications to the previously derived algorithm when the model complexity 
cited above was added. In particular, analytic switching conditions were derived which, under appropriate assumptions, govern optimal transition from one propulsion mode to another for two cases: the case in which engine cycle operations can overlap, and the case in which engine cycle operations are mutually exclusive. The resulting guidance algorithm was implemented in software and exercised extensively. It was found that the approximations associated with the assumed time scale separation employed in this work are reasonable except over the Mach range from roughly 5 to 8 . This phenomenon is due to the very large thrust capability of scramjets in this Mach regime when sized to meet the requirement for ascent to orbit. Very little mass penalty is induced by the resulting inaccuracies in the trajectory over this region because it is traversed rapidly. However, the reduced solution climb paths prove to be unfeasible within this Mach range when subject to the full model dynamics and active trajectory constraints. These difficulties were successfully overcome by accounting for flight path angle and flight path angle rate in construction of the flight path over this Mach range. The resulting algorithm provides the means for rapid near-optimal trajectory generation and propulsion cycle selection over the entire Mach range from take-off to orbit given a realistic nonlinear vehicle model and all pertinent trajectory constraints.

A significant problem area encountered was the lack of a general theory for singularly perturbed systems that are subject to state-variable inequality constraints (Ref. [2]). Such constraints are common to a wide class of flight vehicles but have received little attention in the literature when the dynamic system is singularly perturbed. A study was initiated in this area and it was found that, when the reduced solution lies on a state-variable inequality constraint boundary, the boundary layer trajectories are of finite time in the stretched time scale. The possibility of costate discontinuities at the juncture between constrained and unconstrained arcs makes direct application of existing theory difficult at best. A transformation technique was identified that eliminates some of these difficulties, but at the cost of possibly increased system order and the introduction of singular arcs. Much work remains to be done in this area.

Work on the development of simple, efficient algorithms for prediction of vehicle aerodynamic and propulsive performance continued during the second phase of the program (Ref. [2]). Improvements in the modeling of the hypersonic lifting body module eliminated previous discrepancies between measured and predicted aerodynamic behavior. Several modes of data entry can now be implemented making assessment of a given vehicle configuration very simple. An interactive program mode was devised that makes possible direct and immediate assessment of configuration changes on selected vehicle performance parameters. The algorithms developed in this program are of potential use in applications beyond those originally envisioned.

The first two reporting periods resulted in four conference papers (Ref. [5]-[8]) which discuss most of the results of this research effort. A Ph.D. Dissertation that details the entire effort to date was published in December of 1989 (Ref. [9]). A full-length paper entitled "Rapid Near-Optimal Trajectory Generation for Single-Stage-to-Orbit Airbreathing Vehicles" has also been submitted and accepted for publication in the ALAA Journal of Guidance, Control and Dynamics (Ref. [10]).

There was a funding lag for the period from January to May of 1990 during which no research was conducted. Funding resumed on the first of May.

During the next reporting period, from May 1 to October 31, 1990 efforts were primarily focused upon developing a general understanding of singularly perturbed systems subject to state-variable inequality constraints and also upon developing criteria for the applicability of singular perturbation techniques to flight mechanics problems and in particular to aircraft 
energy climbs (Ref. [3]). As noted in reference [2], singularly perturbed optimal control problems with state-variable inequality constraints can exhibit complex boundary layer phenomena. In particular, the boundary layer transitions associated with such problems can be of finite time when the state constraint is first encountered at the end of the boundary layer transition. The lack of a general theory for treating such systems was identified as a significant research problem.

A cursory look at the problem was completed prior to the submission of the 1989 final report (Ref. [2]). Since that time, considerable progress was made. The results of the effort are detailed in a technical paper that was first presented at the 1990 AIAA GN\&C Conference (Ref. [11]). A revision of the paper has also been accepted for publication in the AIAA Journal of Guidance, Dynamics and Control. The results are summarized as follows.

The established necessary conditions for optimality in nonlinear control problems that involve state-variable inequality constraints were applied to a class of singularly perturbed systems. The distinguishing feature of this class of two-time-scale systems is a transformation of the state-variable inequality constraint, present in the full order problem, to a constraint involving states and controls in the reduced problem. The existence of a nonsingular control solution was assumed. It was of particular interest to construct the zeroth order initial boundary layer solution, or at least and approximation to it, when the reduced solution lies on a state constrain boundary. In the absence of a state constraint, one can take advantage of the fact that the reduced solution serves as an equilibrium point for the boundary layer system. However, it was shown that, when a state constraint is active in the reduced problem, the boundary layer problem can be of finite time in the stretched time variable. Thus, the usual requirement for asymptotic stability of the boundary layer system is not applicable, and can not be used to construct approximate boundary layer solutions. Furthermore, an active state constraint introduced the possibility of discontinuous costate variables at the juncture between constrained and unconstrained arcs.

Various means for treating such problems were investigated. A simple linear example was constructed and used to show that a Valentine transformation can be used to regain smoothness, but with limited advantage. That is, Valentine's transformation can be used to avoid the problems associated with discontinuous costate time histories, but at the expense of introducing a singular arc and discontinuities in the transformed control variable. A second linear example was used to illustrate the exact analytic solution of a simple singularly perturbed problem involving a state variable inequality constraint. The solution includes a "fast" costate discontinuity and a finite-time initial boundary layer transition. A third, but nonlinear, example for which the boundary layer system could not be solved analytically was then constructed. This example was used to illustrate a general feedback strategy that was developed for synthesizing a near-optimal boundary layer transition onto a constrained arc. In this technique, the costate jumps that can occur and the boundary layer final time are used as free parameters in order to satisfy continuity conditions in the state variables at the end of the boundary layer response. The resulting approximation was compared directly with the numerically generated optimal solution. The method proved quite satisfactory when used to construct an approximate solution for this relatively simple nonlinear example, at least for small perturbations away from the reduced solution.

Several problems requiring further attention were identified. For instance, numerical problems were sometimes encountered in the solution process for Example 3 (See Ref. [11]) as time-to-go approached zero (i.e. as the boundary layer transition nears completion). This difficulty did not prevent the generation of an accurate approximation of the optimal solution for the example problem, and further manipulation of Example 3 has 
lead to a completely analytic characterization of the solution. However, the possibility of approaching a singularity should be investigated in a generic setting. The approximation technique being employed depends upon a linearization of the boundary layer system about a non equilibrium point. It does not appear possible to characterize the stability of the approximation for a given set of initial conditions. And though guidance along a constraint boundary will likely be subject to small perturbations only, linearization does introduce the likelihood of control saturation for sufficiently large perturbations. A multiple time scale approach, in which altitude and flight path angle dynamics are examined on separate time scales, could eliminate this dependence on linearization. There is also a question of applicability when atmospheric disturbances lead to a constraint violation.

\section{Progress this reporting period}

During the last reporting period, from November 1, 1990 to August 31, 1991 efforts were again focused upon developing a general understanding of singularly perturbed systems subject to state-variable inequality constraints and also upon developing more stringent criteria for the applicability of singular perturbation techniques to flight mechanics problems and in particular to aircraft energy climbs. Specifically, a systematic approach was devised for naturally identifying the perturbation parameter $\varepsilon$ in a singular perturbation analysis of aircraft optimal guidance and expressing it in terms of original physical problem parameters. The approach, which is based on a nondimensionalization of the equations of motion, can be used to evaluate the appropriateness of forced singular perturbation formulations used in the past for transport and fighter aircraft, and to assess the applicability of energy state approximations and singular perturbation analyses for airbreathing transatmospheric vehicles with hypersonic cruise and orbital capabilities. Furthermore, the approach can easily be extended to asses the possibility of treating the same problems by assuming multiple (more than two) time scale behavior.

The lack of strict criteria for the applicability of singular perturbation techniques to flight mechanics problems served as an incentive for our efforts during the last reporting period. The methods of matched asymptotic analysis in singular perturbation theory are based on the presence of small parameters in the differential equations of motion which give rise to multiple time scale behavior. It has been noted by numerous authors (Ref. [13],[14]) that, in spite of a wide number of papers attesting to the applicability of singular perturbation methods to optimization problems in aircraft flight mechanics, few have been successful in first casting the equations of motion in a singular perturbation form. A few notable exceptions are Refs. [13]-[16]. In Ref. [13] two methods for time scale separation analysis are proposed to identify the proper assignment of state variables to various time scales. These methods are based on forming an estimate of the state variable speeds. In Ref. [14] a rescaling to nondimensional variables is recommended. However, it is noted that the proper scaling transformation is not obvious, even if the time scale separation of the variables is well known from analysis or experience. Both of these papers (and in particular Ref. [13]) provide extensive references to earlier studies which employ so-called forced singular perturbation formulations, in which the perturbation parameter (say $\varepsilon$ ), nominally equal to 1 , is artificially introduced as a book keeping parameter in a formal expansion of the solution about $\varepsilon=0$. In particular, there exists a large number of publications on the optimization of aircraft energy climbs (see for example Refs. [17]-[20]), none of which make any attempt to identify an appropriate perturbation parameter in terms of the original problem parameters. This is particularly disturbing considering the number of years that have passed since such analysis techniques were first introduced in the flight mechanics literature. In any singular perturbation analysis, every attempt should be made to identify 
the perturbation parameter in terms of the original problem parameters (which in general include the boundary conditions) so that the physical process that gives rise to the two time scale behavior is clearly understood. Then, the range of parameter values for which the perturbation analysis is valid can be easily identified. Knowledge of time scale separability present in the system dynamics, and success in exploiting this characteristic to obtain approximate solutions, is not, in itself justification for artificially introducing $\varepsilon$. That is, within the framework of our system of logic it is always possible to have conclusions that are true, which follow from assumptions that are wrong.

Our work has partially rectified this situation by presenting a systematic (albeit still adhoc) approach to nondimensionalize variables in nonlinear optimization problems in flight mechanics. Most of the considerations that were presented apply in other fields as well. Our main motivation for collecting and stating these considerations was to define the thought process by which it is possible to arrive at a suitable scaling of the aircraft energy climb problem. Of particular interest was the assessment of the applicability of energy state approximations and singular perturbation analyses for airbreathing transatmospheric vehicles with hypersonic cruise and orbital capabilities.

The major result of our effort was the demonstration that for energy climbs that take place on a vertical plane the singular perturbation parameter $\varepsilon$ is always equal to the maximum longitudinal loading factor of the vehicle. Two time scale behavior is suggested according to whether $\varepsilon$ is less than one or not. Based on this result it is straightforward to see why singular perturbation methods applied to aircraft performance optimization have worked so well in the past. The maximum longitudinal loading factors associated with the majority of conventional aircraft are either less than one because the aircraft lack very high thrusting capabilities, or because they are restricted to be so for other reasons (structural, comforting, etc.). A few notable exceptions do occur for some modern fighters. This directly suggests that most conventional aircraft can be expected to exhibit two-time-scale behavior for almost any energy climb that they are allowed to perform. This then appears to be the reason for the past success of so many singular perturbation treatments of aircraft energy climbs. The implication for transatmospheric vehicles is rather direct. If we consider such a vehicle as a passenger transport, then, in order to assure passenger comfort it is only natural to impose as a constraint a maximum longitudinal loading factor for the vehicle that is less than one. Our work suggests that such a constraint would imply two-time-scale behavior for any type of energy climb that such a vehicle would be allowed to perform. Therefore, singular perturbation formulations of such maneuvers still appear to be promising, even with all the added complexities that the flight regimes of such vehicles can involve. A paper which reports on our progress has been presented at the 1991 AIAA GN\&C Conference. A copy of the paper is included as an Appendix. 


\section{References}

[1] Calise, A. J., J. E. Corban, and G. A. Flandro, "Trajectory Optimization and Guidance Law Development for National Aerospace Plane Applications," Progress Report for Period December 1, 1988 to June 30, 1989, NASA Grant No. NAG-1922, July 1989.

[2] Corban, J. E., A. J. Calise, and G. A. Flandro, "Rapid Near-Optimal Trajectory Generation and Guidance Law Development for Single-Stage-to-Orbit Airbreathing Vehicles," Progress Report for Period January 1 to December 31, 1989, NASA Grant No. NAG-1-922, January 1990.

[3] Calise, A. J., J. E. Corban, G. A. Flandro, and N. Markopoulos, "Rapid NearOptimal Aerospace Plane Trajectory Generation and Guidance," Progress Report for Period May 1 to October 31, 1990, NASA Grant No. NAG-1-922, November 1990.

[4] Calise, A. J., J. E. Corban, and G. A. Flandro, "Trajectory Optimization and Guidance Law Development for National Aerospace Plane Applications," Final Report for Period July 1, 1987 to November 30, 1988, NASA Grant No. NAG-1784, December 1988.

[5] Calise, A. J., J. E. Corban, and G. A. Flandro, "Trajectory Optimization and Guidance Law Development for National Aerospace Plane Applications," Proceedings of the 1988 ACC, Vol. 2, pp. 1406-1411, Atlanta, GA, June 15-17.

[6] Corban, J. E., Calise, A. J., and Flandro, G. A., "Trajectory Optimization and Guidance Law Development for Transatmospheric Vehicles," Proceeding of the 1989 IEEE International Conference on Control and Applications (ICCON), April 3-6, Jerusalem, Israel.

[7] Corban, J. E., A. J. Calise, and G. A. Flandro, "A Real-Time Guidance Algorithm for Aerospace Plane Optimal Ascent to Low Earth Orbit," Proceedings of the 1989 ACC, Vol. 3, June 21-23, Pittsburgh, PA, pp. 2475-2481.

[8] Corban, J. E., A. J. Calise, and G. A. Flandro, "Optimal Guidance and Propulsion Control for Transatmospheric Vehicles," Presented at the 1989 AIAA GN\&C Conference, August 14-16, Boston, MA, available as AIAA Paper 89-3617.

[8a] Calise, A. J., and J. E. Corban, "Optimal Control of Singularly Perturbed Nonlinear Systems with State Variable Inequality Constraints," Presented at the IFAC Workshop on Singular Perturbations and Asymptotic Methods in Systems and Control, Boston, MA, August 17-18, 1989.

[9] Corban, J. E., "Real-Time Guidance and Propulsion Control for Single-Stage-toOrbit Airbreathing Vehicles," Ph.D. Dissertation, The Georgia Institute of Technology, December 1989.

[10] Corban, J. E., A. J. Calise, and G. A. Flandro, "Rapid Near-Optimal Aerospace Plane Trajectory Generation and Guidance," Accepted for Publication in the AIAA Journal of Guidance, Dynamics and Control. 
[11] Calise, A. J., and J. E. Corban, "Optimal Control of Singularly Perturbed Nonlinear Systems with State-Variable Inequality Constraints," Proceedings of the 1990 AIAA Guidance, Navigation and Control Conference, August 20-22, Portland, OR, also accepted for publication in the AIAA Journal of Guidance, Dynamics and Control.

[12] Calise A. J., N. Markopoulos, and J. E. Corban, "Nondimensional Forms for Singular Perturbation Analyses of Aircraft Energy Climbs," Presented at the 1991 AIAA GN\&C Conference, August 11-14, New Orleans, LA, available as AIAA Paper 91-2640.

[13] Ardema, M. D., "Separation of Time Scales in Aircraft Trajectory Optimization," AIAA Journal of Guidance and Control, Vol. 8, No. 2, March-April 1985.

[14] Shinar, J., "On Applications of Singular Perturbation Techniques in Nonlinear Optimal Control," Automatica, Vol. 19, No. 2, 1983.

[15] Breakwell, J. V., "Optimal Flight-Path Angle Transitions in Minimum-Time Airplane Climbs," Journal of Aircraft, Vol. 14, August 1977.

[16] Calise, A. J., "Optimal Thrust Control with Proportional Navigation Guidance," AIAA Journal of Guidance and Control, Vol. 3, No. 4, July-August 1980.

[17] Kelley, H. J. and Edelbaum, T. N., "Energy Climbs, Energy Turns, and Asymptotic Expansions," Journal of Aircraft, Vol. 7, Jan-Feb 1970.

[18] Ardema, M. D., "Solution of the Minimum Time-to-Climb Problem by Matched Asymptotic Expansions," AIAA Journal, Vol. 14, No. 7, July 1976.

[19] Calise, A. J., "Extended Energy Management Methods for Flight Performance Optimization," AIAA Journal, Vol. 15, No. 3, March 1977.

[20] Calise, A. J., "Singular Perturbation Techniques for On-Line Optimal Flight-Path Control," AIAA Journal of Guidance and Control, Vol. 4, No. 4, July-August 1981.

[21] Corban, J. E., Calise, A. J., and Flandro, G. A., "Rapid Near-Optimal Trajectory Generation for Single-Stage-to-Orbit Airbreathing Vehicles," To appear in the AIAA Journal of Guidance, Control and Dynamics. 


\section{Appendix}

Nondimensional Forms for Singular Perturbation Analysis of Aircraft Energy Climbs 


\title{
NONDIMENSIONAL ForMS FOR SINGULAR Perturbation Analyses of Aircraft ENERgy ClimbS
}

\author{
A. J. Calise, N. Markopoulos and J. E. Corban \\ Aerospace Engineering Department \\ Georgia Institute of Technology, Atlanta, Georgia
}

Presented at the AIAA Guidance, Navigation and Control Conference

New Orleans, Luisiana, August 12-14, 1991 


\begin{abstract}
This paper proposes a systematic approach for identifying the perturbation parameter in singular perturbation analysis of aircraft optimal guidance, and in particular considers a family of problems related to aircraft energy climbs. It is first shown that for energy climbs that take place on a vertical plane the singular perturbation parameter can always be taken to be the maximum allowed longitudinal loading factor of the vehicle. Two time scale behavior is suggested according to whether this parameter is sufficiently less than one. The approach, which is based on a nondimensionalization of the equations of motion, is then used to evaluate the appropriateness of forced singular perturbation formulations used in the past for transport and fighter aircraft, and to assess the applicability of energy state approximations and singular perturbation analysis for airbreathing transatmospheric vehicles with hypersonic cruise and orbital capabilities.
\end{abstract}

\title{
Introduction
}

The methods of matched asymptotic analysis in singular perturbation theory are based on the presence of small parameters in the differential equations of motion which give rise to multiple time scale behavior. It has been noted by several authors 1,2 that, in spite of a wide number of papers attesting to the applicability of singular perturbation methods to optimization problems in aircraft flight mechanics, few have been successful in first casting the equations of motion in a singular perturbation form. A few notable exceptions are Refs. 1-4. In Ref. 1 two methods for time scale separation analysis are proposed to identify the proper assignment of state variables to various time scales. These methods are based on forming an estimate of the state variable speeds. In Ref. 2 a rescaling to nondimensional variables is recommended. However, it is noted that the proper scaling transformation is not obvious, even if the time scale separation of the variables is well known from analysis or experience. Both of these papers (and in particular Ref. 1) provide extensive references to earlier studies which employ so-called forced singular perturbation formulations, in which the perturbation parameter (say $\varepsilon$ ), nominally equal to 1 , is artificially introduced as a book keeping parameter in a formal expansion of the solution about $\varepsilon=0$. In particular, there exists a large number of publications on the optimization of aircraft energy climbs (see 
for example References 5-8), none of which make any attempt to identify an appropriate perturbation parameter in terms of the original problem parameters. This is particularly disturbing considering the number of years that have passed since such analysis techniques were first introduced in the flight mechanics literature. In any singular perturbation analysis, every attempt should be made to identify the perturbation parameter in terms of the original problem parameters (which in general include the boundary conditions) so that the physical process that gives rise to the two time scale behavior is clearly understood. Then, the range of parameter values for which the perturbation analysis is valid can be easily identified. Knowledge of time scale separability present in the system dynamics, and success in exploiting this characteristic to obtain approximate solutions, is not, in itself justification for artificially introducing $\varepsilon$. That is, within the framework of our system of logic it is always possible to have conclusions that are true, which follow from assumptions that are wrong.

In this note we attempt to partially rectify this situation by presenting a systematic (albeit still adhoc) approach to nondimensionalize variables in nonlinear optimization problems in flight mechanics. Most of the considerations that are presented apply in other fields as well. Our main motivation for collecting and stating these considerations is to define the thought process by which it is possible to arrive at a suitable scaling of the aircraft energy climb problem. Of particular interest is an assessment of the applicability of energy state approximations and singular perturbation analysis for airbreathing transatmospheric vehicles with hypersonic cruise and orbital capabilities.

\section{Subsonic-Supersonic Regimes, Flat Earth Approximation}

Consider atmospheric flight of a conventional aircraft, viewed as a point mass, in a vertical plane over a flat Earth. The equations governing such flight can be reduced to a three-state model in: mass specific energy $E$, flight path angle $\gamma$, and altitude $h$. The vehicle mass, $m$, is assumed to be constant. The equations are:

$$
\begin{aligned}
& \frac{d E}{d t}=\frac{V(T-D)}{m} \\
& \frac{d \gamma}{d t}=\left(\frac{L}{m V}\right)-\left(\frac{g \cos \gamma}{V}\right)
\end{aligned}
$$




$$
\frac{\mathrm{dh}}{\mathrm{dt}}=\mathrm{V} \sin \gamma
$$

where $\mathrm{L}, \mathrm{D}$ and $\mathrm{g}$ denote the lift, the drag and the (constant) gravitational acceleration. It is assumed that the atmosphere is stationary, and that the thrust, $T$, is directed along the flight path. The specific energy (mechanical energy per unit mass of the vehicle) $\mathrm{E}$ and the speed $\mathrm{V}$ are related by

$$
E=\frac{V^{2}}{2}+g h
$$

and $E$ rather than $V$ has been employed as a state variable.

In many of the earlier singular perturbation studies the traditional way of writing down Eqs. (2) and (3) was:

$$
\begin{aligned}
& \varepsilon \frac{\mathrm{d} \gamma}{\mathrm{dt}}=\left(\frac{\mathrm{L}}{\mathrm{mV}}\right)-\left(\frac{\mathrm{g} \cos \gamma}{\mathrm{V}}\right) \\
& \varepsilon \frac{\mathrm{dh}}{\mathrm{dt}}=\mathrm{V} \sin \gamma
\end{aligned}
$$

that is, by artificially introducing a parameter $\varepsilon$ and then stating that its nominal value was equal to 1 . Since our main purpose in the present paper is to avoid such an artificial introduction at the outset, Eqs. (5) and (6) will serve only as a guide for the natural introduction of $\varepsilon$.

\section{Nondimensional form}

The first step is to put Eqs. (1) through (3) in nondimensional form. To this end we define the set $S$

$$
S \equiv\left\{t_{0}, E_{0}, h_{0}, V_{0}, T_{0}, D_{0}, L_{0}\right\}
$$

The elements of the set $S$ are at this point arbitrary positive quantities, and the only restriction that we impose upon them is that:

$t_{0}$ has dimensions of time

$E_{0}$ has dimensions of energy per unit mass

$h_{0}$ has dimensions of lenght

$\mathrm{V}_{0}$ has dimensions of speed 
$T_{0}, D_{0}$, and $L_{0}$ have dimensions of force

Using the elements of $S$ to define the nondimensional quantities:

$$
\begin{aligned}
& \mathrm{t}=\frac{\mathrm{t}}{\mathrm{t}_{0}} ; \mathrm{E}=\frac{\mathrm{E}}{\mathrm{E}_{0}} ; \mathrm{h}=\frac{\mathrm{h}}{\mathrm{h}_{0}} ; \mathrm{V}=\frac{\mathrm{V}}{\mathrm{V}_{0}} \\
& \mathrm{~T}=\frac{\mathrm{T}}{\mathrm{T}_{0}} ; \mathrm{D}=\frac{\mathrm{D}}{\mathrm{D}_{0}} ; \mathrm{L}=\frac{\mathrm{L}}{\mathrm{L}_{0}}
\end{aligned}
$$

Eqs. (1) through (3) can be put into the following nondimensional form:

$$
\begin{aligned}
\frac{d E}{d t} & =V\left(T T_{0}-D D_{0}\right)\left(\frac{t_{0} V_{0}}{E_{0} m}\right) \\
\frac{d \gamma}{d t} & =\left(\frac{L}{V}\right)\left(\frac{L_{0} t_{0}}{m V_{0}}\right)-\left(\frac{\cos \gamma}{V}\right)\left(\frac{g t_{0}}{V_{0}}\right) \\
\frac{d h}{d t} & =\left(\frac{V_{0} t_{0}}{h_{0}}\right) V \sin \gamma
\end{aligned}
$$

The goal is now to put Eqs. (10) through (12) in the traditional singular perturbation format. We thus multiply both sides of Eqs. (11) and (12) by $\left(\mathrm{h}_{0} / \mathrm{V}_{0} \mathrm{t}_{0}\right)$. This results in:

$$
\begin{aligned}
& \left(\frac{h_{0}}{V_{0} t_{0}}\right) \frac{d \gamma}{d t}=\left(\frac{L}{V}\right)\left(\frac{L_{0} h_{0}}{m V_{0}^{2}}\right)-\left(\frac{\cos \gamma}{V}\right)\left(\frac{g h_{0}}{V_{0}^{2}}\right) \\
& \left(\frac{h_{0}}{V_{0} t_{0}}\right) \frac{d h}{d t}=V \sin \dot{\gamma}
\end{aligned}
$$

Comparing the set of Eqs. (10), (13) and (14) with the set (1), (5) and (6), it is evident that we can make the two sets similar by imposing the following four conditions on the elements of the set $S$ :

$$
\begin{aligned}
& \mathrm{T}_{0}=\mathrm{D}_{0} \\
& \frac{\mathrm{T}_{0} \mathrm{t}_{0} \mathrm{~V}_{0}}{\mathrm{E}_{0} \mathrm{~m}}=1 \\
& \frac{\mathrm{L}_{0} \mathrm{~h}_{0}}{\mathrm{~m} \mathrm{V_{0 } ^ { 2 }}}=1
\end{aligned}
$$




$$
\frac{g h_{0}}{V_{0}^{2}}=1
$$

If we define $\varepsilon$ as

$$
\varepsilon \equiv \frac{\mathrm{h}_{0}}{\mathrm{~V}_{0} \mathrm{t}_{0}}
$$

then, Eqs. (10), (13) and (14) assume the form:

$$
\begin{aligned}
\frac{d E}{d t} & =V(T-D) \\
\varepsilon \frac{d \gamma}{d t} & =\left(\frac{L-\cos \gamma}{V}\right) \\
\varepsilon \frac{d h}{d t} & =V \sin \gamma
\end{aligned}
$$

To summarize, it was shown in the present section that it is possible to introduce a parameter $\varepsilon$ naturally into the equations of motion (Eqs. (1) - (3)) by first introducing a set of arbitrary positive quantities S (see Eq. (7)) to scale the variables of interest, and then by imposing four conditions (Eqs. (15) - (18)) on these quantities so that the resulting nondimensional equations assume the traditional singular perturbation form (Eqs. (20) - (22)). Note that only one of the arbitrary quantities in $S$ is uniquely determined at this point. Combining Eqs. (17) and (18) it follows that

$$
\mathrm{L}_{0}=\mathrm{mg}
$$

\section{Specifying a particular nondimensional form}

As shown in the previous section, only four conditions are imposed on the seven elements of the set $S$ in transforming the equations of motion to the traditional singular perturbation format. This means that we can specify three of the elements of $S$ to fit our convenience and then determine the remaining four using Eqs. (15)-(18). The first conclusion therefore is that in general the value of $\varepsilon$ is quite arbitrary. For example, by choosing $h_{0}, V_{0}$ and $t_{0}$ in two different ways $\varepsilon$ can be made arbitrarily small or large. The separability of the time scales on the other hand is a property of the system and not of the

particular nondimensional form of the equations of motion that is chosen. We therefore expect that if the 
system does indeed have this property it will exhibit it no matter what the actual value of $\varepsilon$ is. This is precisely the reason for the success of so many singular perturbation treatments of the past in which $\varepsilon$ was introduced artificially and its nominal value was said to be fixed at one.

Although there is no unique way of specifying a particular nondimensional form of the equations of motion, we will now argue that there is one that results in additional physical insight. First, in order to maintain the relationship in Eq. (4) in the transformed variables, a fifth condition is introduced

$$
\mathrm{E}_{0}=\mathrm{gh}_{0}
$$

which together with Eq. (18) gives

$$
E=\frac{V^{2}}{2}+h
$$

Using Eqs. (16) and (24) in Eq. (19) it follows that

$$
\varepsilon=\frac{T_{0}}{\mathrm{mg}}
$$

It is now evident that only two among the seven elements of the set $\mathbf{S}$ need to be specified. Then, the five conditions, Eqs. (15) - (18) and (24), uniquely determine the remaining elements.

Eq. (26) implies that $\varepsilon$ depends only on $\mathrm{T}_{0}$ and is independent of the value of the remaining elements of $S$. The question therefore arises naturally as to whether there is a particular choice of $T_{0}$ for which the resulting value of $\varepsilon$ can be used as a strict criterion for the applicability of a singular perturbation analysis to Eqs. (20)-(22). The answer to this question is negative because, in a given time interval, it is the relative magnitudes of the three quantities

$$
\frac{\mathrm{dE}}{\mathrm{dt}}, \frac{\mathrm{d} \gamma}{\mathrm{dt}}, \frac{\mathrm{dh}}{\mathrm{dt}}
$$

and the boundary conditions of interest that determine the validity of a singular perturbation analysis. Specifically, for an aircraft to exhibit the well-known two-time-scale behavior in a given time interval, it is necessary that:

$$
\frac{\mathrm{dE}}{\mathrm{dt}}<<\frac{\mathrm{d} \gamma}{\mathrm{dt}}
$$




$$
\frac{d E}{d t} \ll<\frac{d h}{d t}
$$

in that interval, and that the required change in $E$ is sufficiently large to permit the boundary layer responses in $\mathrm{h}$ and $\gamma$ to reach their equilibrium values. Hence, the very question is whether or not the net change in $E$ during the boundary layer response is sufficiently small to permit approximating $E$ as a constant (to zero order in $\varepsilon$ ) in the boundary layer analysis. In addition, we are interested in knowing if this two-time-scale property is a consequence of the inherent dynamics of the aircraft, and not a consequence of using a high gain control solution for $L$. Therefore, we assume that the $L$ resulting from the boundary layer analysis is of order one in Eq. (21).

Under the above assumptions, there is a choice for $T_{0}$ for which the value of $\varepsilon$ can be used as a measure for the existence of time intervals in which two-time-scale behavior is exhibited. If the choice of $T_{0}$ is such that $d E / d t$ is at most of the same order of magnitude as $\varepsilon d \gamma / d t$ and $\varepsilon d h / d t$, then, a value of $\varepsilon$ sufficiently less than one indicates the possible existence of such intervals. By suitably choosing $V_{0}$ we can restrict $V$ to be of order one. Then, for the choice

$$
\mathrm{T}_{0}=(\mathrm{T}-\mathrm{D})_{\max }
$$

$d E / d t$ is of order one, and both $d \gamma / d t$ and $d h / d t$ are of order $1 / \varepsilon$. For this choice of $T_{0}, \varepsilon$ is given by

$$
\varepsilon=\frac{(T-D)_{\max }}{m g}
$$

and is equal to the maximum longitudinal loading factor of the vehicle.

Note that Eq. (30) actually represents an upper bound for $\varepsilon$ since it is obtained by selecting the flight condition where the difference between thrust and drag, T-D, reaches a maximum. The logical choice for $\mathrm{V}_{0}$ is the speed at this flight condition. One can also adopt the viewpoint of evaluating $\varepsilon$ along the energy climb path that results from a reduced solution. The value of $\varepsilon$ as a function of $E$ can then be used as a measure to distinguish energy levels where a singular perturbation analysis may be appropriate from other levels where it may not be valid.

It is interesting to note that a good deal can be anticipated from Eq. (30) for conventional aircraft without exact numerical evaluation: (T-D) $\max$ divided by $\mathrm{mg}$ is approximately equal to $\sin \gamma_{\max }$ where 
$\gamma_{\max }$ is the maximum climb angle that can be maintained at a given energy level without loss of airspeed. It follows therefore that $\varepsilon<1$ for all such aircraft types. For transport aircraft $\sin \gamma_{\max }$ is approximately 0.1 , while for fighter aircraft $\sin \gamma_{\max }$ is approximately 0.8 . This suggests that the forced singular perturbation analysis used in the past studies of optimal aircraft trajectories is.valid for most conventional subsonic and supersonic aircraft.

Eq. (30) can also be used to estimate $\varepsilon$ in terms of the quantities $(T / \mathrm{mg})_{\max }$ and $(\mathrm{L} / \mathrm{D})_{\max }$ for a given aircraft. Since $\mathrm{L}$ is less than or equal to $\mathrm{mg}$ along the energy climb path, it follows that a second upper bound for $\varepsilon$ is given by

$$
\varepsilon<\varepsilon_{\mathrm{UB}}
$$

where $\varepsilon_{U B}$ is defined as

$$
\varepsilon_{\mathrm{UB}} \equiv\left[(\mathrm{T} / \mathrm{mg})_{\max }-1 /(\mathrm{L} / \mathrm{D})_{\max }\right]
$$

Estimates of $\varepsilon_{\mathrm{UB}}$ are given in Table 1 .

\section{Table 1}

Estimation of $\varepsilon_{\mathrm{UB}}$ based on Eq. (32)

$\begin{array}{lcc}\text { Parameter } & \text { Transports } & \text { Fighters } \\ * * * * * * * * * * * * * * * * * * * * * * * * * * * * * * * * * * * * * * * * * * * * * * * * * * * * * \\ (\mathrm{~T} / \mathrm{mg})_{\max } & 0.25 & 0.90 \\ (\mathrm{~L} / \mathrm{D})_{\max } & 13-15 & 4-7 \\ \varepsilon_{\mathrm{UB}} & 0.17-0.18 & 0.65-0.76\end{array}$

\section{Hypersonic Regime}

Consider the flight of a hypersonic and possibly transatmospheric vehicle, viewed as a point mass, in a vertical plane over a spherical non-rotating Earth. The equations governing such flight can be reduced to a four-state model in $\mathrm{E}, \mathrm{m}, \gamma$ and radial distance from the center of the Earth, $r$. The equations are:

$$
\frac{\mathrm{dE}}{\mathrm{dt}}=\frac{\mathrm{V}(\eta \mathrm{T}-\mathrm{D})}{\mathrm{m}}
$$




$$
\begin{aligned}
& \frac{\mathrm{dm}}{\mathrm{dt}}=-\mathrm{f}(\mathrm{r}, \mathrm{V}, \eta) \\
& \frac{\mathrm{d} \gamma}{\mathrm{dt}}=\left(\frac{\mathrm{L}}{\mathrm{mV}}\right)-\left(\frac{\mu \cos \gamma}{\mathrm{Vr}^{2}}\right)+\left(\frac{\mathrm{V} \cos \gamma}{\mathrm{r}}\right) \\
& \frac{\mathrm{dr}}{\mathrm{dt}}=\mathrm{V} \sin \gamma
\end{aligned}
$$

where $\mathrm{T}$ is the maximum available thrust at a given speed and altitude. The control variables are $\mathrm{L}$ and $\eta$, where $0<\eta<1$ is introduced as a nondimensional throttling variable. $E$ and $V$ are now related by

$$
E=\frac{V^{2}}{2}-\frac{\mu}{r}
$$

Note again that in earlier singular perturbation studies ${ }^{9}$, the traditional way of writing down Eqs. (35) and (36) was:

$$
\begin{aligned}
& \varepsilon \frac{\mathrm{d} \gamma}{\mathrm{dt}}=\left(\frac{\mathrm{L}}{\mathrm{mV}}\right)-\left(\frac{\mu \cos \gamma}{\mathrm{V} \mathrm{r}^{2}}\right)+\left(\frac{\mathrm{V} \cos \gamma}{\mathrm{r}}\right) \\
& \varepsilon \frac{\mathrm{dr}}{\mathrm{dt}}=\mathrm{V} \sin \gamma
\end{aligned}
$$

that is, by artificially introducing a parameter $\varepsilon$ and then stating that its nominal value was equal to 1 . Again, in order to avoid this artificial introduction, Eqs. (38) and (39) will serve only as a guide for the natural introduction of $\varepsilon$.

\section{Nondimensional Form}

In order to put Eqs. (33) through (36) in nondimensional form we now define the set of arbitrary positive quantities

$$
\mathrm{S} \equiv\left\{\mathrm{t}_{0}, \mathrm{E}_{0}, \mathrm{~m}_{0}, \mathrm{r}_{0}, \mathrm{~V}_{0}, \mathrm{f}_{0}, \mathrm{~T}_{0}, \mathrm{D}_{0}, \mathrm{~L}_{0}\right\}
$$

and impose the restrictions that:

$t_{0}$ has dimensions of time

$\mathrm{E}_{0}$ has dimensions of energy per unit mass

$\mathrm{m}_{0}$ has dimensions of mass

$\tau_{0}$ has dimensions of lenght 
$\mathrm{V}_{0}$ has dimensions of speed

$f_{0}$ has dimensions of mass per unit time

$\mathrm{T}_{0}, \mathrm{D}_{0}$, and $\mathrm{L}_{0}$ have dimensions of force

Using the elements of $S$ to define the nondimensional quantities:

$$
\begin{aligned}
& t=\frac{t}{t_{0}} ; E=\frac{E}{E_{0}} ; m=\frac{m}{m_{0}} ; r=\frac{r}{r_{0}} ; V=\frac{V}{V_{0}} \\
& \mathrm{f}=\frac{f}{f_{0}} ; T=\frac{T}{T_{0}} ; D=\frac{D}{D_{0}} ; L=\frac{L}{L_{0}}
\end{aligned}
$$

Eqs. (33) through (36) can be put into the following nondimensional form:

$$
\begin{aligned}
& \frac{d E}{d t}=\frac{V\left(\eta T T_{0}-D D_{0}\right)}{m}\left(\frac{t_{0} V_{0}}{E_{0} m_{0}}\right) \\
& \frac{d m}{d t}=-\left(\frac{f_{0} t_{0}}{m_{0}}\right) f(r, V, \eta) \\
& \frac{d \gamma}{d t}=\left(\frac{L}{m V}\right)\left(\frac{L_{0} t_{0}}{m_{0} V_{0}}\right)-\left(\frac{\cos \gamma}{V r^{2}}\right)\left(\frac{\mu t_{0}}{V_{0} r_{0}^{2}}\right)+\left(\frac{V \cos \gamma}{r}\right)\left(\frac{V_{0} t_{0}}{r_{0}}\right) \\
& \frac{d r}{d t}=\left(\frac{V_{0} t_{0}}{r_{0}}\right) V \sin \gamma
\end{aligned}
$$

In order to put Eqs. (43) through (46) in the traditional singular perturbation format, we multiply both sides of Eqs. (45) and (46) by $\left(r_{0} / V_{0} t_{0}\right)$. This results in:

$$
\begin{aligned}
& \left(\frac{\mathrm{r}_{0}}{\mathrm{~V}_{0} \mathrm{t}_{0}}\right) \frac{\mathrm{d} \gamma}{\mathrm{dt}}=\left(\frac{\mathrm{L}}{\mathrm{mV}}\right)\left(\frac{\mathrm{L}_{0} \mathrm{r}_{0}}{\mathrm{~m}_{0} \mathrm{~V}_{0}^{2}}\right)-\left(\frac{\cos \gamma}{V \mathrm{r}^{2}}\right)\left(\frac{\mu}{\mathrm{V}_{0}^{2} \mathrm{r}_{0}}\right)+\left(\frac{\mathrm{V} \cos \gamma}{\mathrm{r}}\right) \\
& \left(\frac{\mathrm{r}_{0}}{\mathrm{~V}_{0} \mathrm{t}_{0}}\right) \frac{\mathrm{dr}}{\mathrm{dt}}=\mathrm{V} \sin \gamma
\end{aligned}
$$

Comparing the set of Eqs. (43), (44), (47) and (48) with the set (33), (34), (38) and (39) results in the following five conditions on the elements of the set $\mathrm{S}$ :

$$
\mathrm{T}_{0}=\mathrm{D}_{0}
$$




$$
\begin{aligned}
& \frac{\mathrm{T}_{0} \mathrm{t}_{0} \mathrm{~V}_{0}}{\mathrm{E}_{0} \mathrm{~m}_{0}}=1 \\
& \frac{\mathrm{f}_{0} \mathrm{t}_{0}}{\mathrm{~m}_{0}}=1 \\
& \frac{\mathrm{L}_{0} \mathrm{r}_{0}}{\mathrm{~m}_{0} \mathrm{~V}_{0}^{2}}=1 \\
& \frac{\mu}{\mathrm{V}_{0}^{2} \mathrm{r}_{0}}=1
\end{aligned}
$$

By defining

$$
\varepsilon \equiv \frac{\mathrm{r}_{0}}{\mathrm{~V}_{0} \mathrm{t}_{0}}
$$

Eqs. (43), (44), (47) and (48) assume the traditional singular perturbation form:

$$
\begin{aligned}
\frac{d E}{d t} & =\frac{V(\eta T-D)}{m} \\
\frac{d m}{d t} & =-f(r, V, \eta) \\
\varepsilon \frac{d \gamma}{d t} & =\left(\frac{L}{m V}\right)-\left(\frac{\cos \gamma}{V r^{2}}\right)+\left(\frac{V \cos \gamma}{r}\right) \\
\varepsilon \frac{d r}{d t} & =V \sin \gamma
\end{aligned}
$$

\section{Specifying a particular nondimensional form}

For the hypersonic case only five conditions on the nine elements of the set $\mathrm{S}$ are needed in order to put the equations of motion in the traditional singular perturbation format. Thus, we can specify four of the elements of $S$ to fit our convenience and then determine the remaining five using Eqs. (49) - (53).

Again, in order to maintain the relationship in Eq. (37) in the transformed variables, a sixth condition is introduced 


$$
E_{0}=\frac{\mu}{r_{0}}
$$

which together with Eq. (53) gives

$$
E=\frac{V^{2}}{2}-\frac{1}{r}
$$

If we think of $r_{0}$ as a radial distance, then Eq. (53) restricts $V_{0}$ to be the circular orbital speed at $r_{0}$. Similarly, Eq. (52) restricts $\mathrm{L}_{0}$ to be the centrifugal force that a point mass $\mathrm{m}_{0}$ would experience in a circular orbit at $r_{0}$. Using Eqs. (50), (53), and (59) we have

$$
\varepsilon=\frac{\mathrm{T}_{0} \mathrm{r}_{0}^{2}}{\mu \mathrm{m}_{0}}
$$

Hence, by picking three among the nine elements of the set $S$ arbitrarily, the six conditions Eqs. (49) (53), and (59) uniquely determine the remaining elements.

The question arises again as to whether there is a particular choice for these three elements for which the resulting value of $\varepsilon$ can be used as a measure for the applicability of a singular perturbation analysis to Eqs. (55)-(58). The right-hand-side of Eqs. (55) and (58) can be made of the same order of magnitude by choosing the ratio $\mathrm{T}_{0} / \mathrm{m}_{0}$ as

$$
\frac{\mathrm{T}_{0}}{\mathrm{~m}_{0}}=\left(\frac{\eta \mathrm{T}-\mathrm{D}}{\mathrm{m}}\right)_{\max }
$$

Choosing $r_{0}$ as the sea level radius $r_{S L}, r$ and $V$ are of order one. Also, for these choices of $T_{0} / m_{0}$ and $r_{0}, d E / d t$ is of order one, and both $d \gamma / d t$ and $d r / d t$ are of order $1 / \varepsilon$. By choosing $f_{0}$ as the value of $f$ at the flight condition where the ratio $(\eta T-D) / m$ is a maximum, $\mathrm{dm} / \mathrm{dt}$ can also be made of order one. With the above choices of $T_{0} / m_{0}, r_{0}$, and $f_{0}$

$$
\varepsilon=\left(\frac{\mathrm{r}_{\mathrm{SL}}^{2}}{\mu}\right)\left(\frac{\eta \mathrm{T}-\mathrm{D}}{\mathrm{m}}\right)_{\max }
$$

The right-hand-side of Eq. (63) is the maximum longitudinal loading factor of the vehicle in units of sea-level g's, and actually represents an upper bound for $\varepsilon$ since it is obtained by selecting the flight condition where $(\eta T-D) / m$ reaches a maximum. One can again adopt the viewpoint of evaluating $\varepsilon$ along the energy climb path that results from the reduced solution. The value of $\varepsilon$ as a function of $E$ can then be 
used as a measure to distinguish energy levels where a singular perturbation analysis may be appropriate, from other levels where it may not be valid.

A hypersonic flight vehicle employing an airbreathing propulsion system and sized for acceleration to orbital velocity necessarily employs a multimode propulsion system. An example might include turbojet, ramjet, scramjet, and rocket modes. Each mode of propulsion can be characterized by a corresponding $\varepsilon$. Current models of this vehicle type exhibit large values of excess thrust at low hypersonic Mach numbers. In fact, Eq. (63) will produce an $\varepsilon$ that is greater than one over such flight phases. Experience with hypersonic vehicle dynamics reported in Ref. 10 indeed suggests that the assumed time scale separation is not valid in these phases. However, over the majority of the trajectory, Eq. (63) results in an $\varepsilon$ that is less than one just as in the Flat Earth, Subsonic-Supersonic case.

\section{Numerical validation}

It was shown in the preceding sections that for aircraft energy climbs that take place in a vertical plane, the singular perturbation parameter $\varepsilon$ can always be identified as the maximum longitudinal loading factor of the vehicle, measured in units of sea-level g's. In order to further explore the implications of this result, numerical evaluations of $\varepsilon$ will be presented in this section for several types of vehicles.

The idea that the authors would like to introduce at this point is that in general, for a given aircraft, it may be sufficient to evaluate an upper bound for $\varepsilon$, valid for the entire envelope, in order to get a hint for the possible two-time-scale behavior of the aircraft in question. If the resulting value of this upper bound is less than one, then, two-time-scale behavior is implied for any energy climb that the aircraft is allowed to perform. If however the resulting value of the upper bound turns out to be greater than one, then no conclusion can be drawn. The way to proceed in this latter case would be to evaluate a less conservative upper bound for $\varepsilon$ and apply the same reasoning. As it turns out, the less conservative the upper bound, the more work one has to perform in order to evaluate it. If all the upper bounds for $\varepsilon$, evaluated for the entire envelope fail to yield any conclusions, the reasonable thing to do next is to evaluate $\varepsilon$ as a function of the energy $E$ using all the assumptions made in the evaluation of reduced 
solutions in aircraft energy climbs $(\gamma=0, \mathrm{~L}=\mathrm{mg}$ etc. $)$. By evaluating in this sense, and at each energy level the absolute maximum value of the longitudinal loading factor we obtain a curve $C$ on the $\varepsilon-E$ plane. The interesting properties of this curve are that for a given aircraft it need only be constructed once and that it lies above all other curves that may be evaluated similarly, but along the reduced solutions corresponding to specific problems. In other words, points on curve $C$ represent upper bounds for $\varepsilon$ at the corresponding energy levels. The portions therefore of curve $C$ where $\varepsilon$ is less than one immediately show the energy levels where two-time-scale behavior (boundary layer transitions along constant $E$ ) can be expected. If there are any portions of curve $C$ where $\varepsilon$ is greater than one, then no conclusions can be drawn as to the possible two-time-scale behavior at the corresponding energy levels. In the latter case one has again to evaluate a less conservative upper bound for $\varepsilon$ at these energy levels. Such less and less conservative upper bounds would of course eventually lead to the maximum value of the longitudinal loading factor evaluated as a function of $\mathrm{E}$ along the reduced solution corresponding to a specific problem.

It should be clear now that if we are interested in the possible two-time-scale behavior of a vehicle along a particular trajectory (corresponding to a specific problem) then the least conservative upper bound for $\varepsilon$ would be the maximum longitudinal loading factor encountered along that (exact) trajectory. Calculating this upper bound would not be very useful since it would require the actual computation of the trajectory first. The idea presented above suggests that there may be a hope of avoiding this by starting with a more conservative upper bound, and proceeding with less and less conservative upper bounds.

In order to demonstrate the above ideas in practice, numerical evaluations of $\varepsilon$ are presented in Figs. 1-8 for four types of vehicles. For each type there is one plot showing the variation of the maximum longitudinal loading factor of the vehicle with energy $\mathrm{E}$, and one or more plots showing the variation of the longitudinal loading factor with $\mathrm{E}$ along the reduced solution corresponding to a specific optimization problem.

Figs. 1 and 2 show the results for an F- 8 fighter ${ }^{10}$. The two optimization problems considered for this case were minimum time to a specified energy and minimum time to a specified downrange position. 
The reduced solutions corresponding to these problems are obtained by maximizing (with respect to $\mathrm{V}$ ) at each energy level the quantities (T-D)V for the former and $[(T-D) V] /\left(V_{0}-\mathrm{V}\right)$ for the latter, where $V_{0}$ is the maximum possible cruising speed of the aircraft and $\mathrm{D}$ is calculated at $\mathrm{L}=\mathrm{mg}$. Fig. 1 shows the actual paths in the envelope corresponding to these reduced solutions and to the maximum longitudinal loading factor of F-8. Fig. 2 shows the results for $\varepsilon$ evaluated along these climb paths. Since the maximum longitudinal loading factor of F-8 stays always below one in Fig. 2, it is reasonable to assume that for a any optimization problem, if the required energy gain is sufficient, the transitions to the reduced solution will take place at nearly constant $E$, exhibiting the well known boundary layer structure.

Figs. 3 and 4 show similar results for an F-15 fighter ${ }^{11}$. The two optimization problems considered in this case were again minimum time to a specified energy and minimum time to a specified downrange position. A maximum dynamic pressure constraint of $1500 \mathrm{lbf}$ per square feet is imposed on the climb paths for this case. Due to the large thrust to weight ratio of F-15, the $\varepsilon$ levels in Fig. 4 are much higher than the ones corresponding to F-8 (compare with Fig. 2). In particular there is a small region at low energy where $\varepsilon$ exceeds one, implying that two time scale separation at these energy levels may not be appropriate for the above two optimization problems.

Figs. 5 and 6 show the results for a conventional transport ${ }^{12}$. In this case however, the two optimization problems considered were minimum fuel to a specified energy and minimum fuel to a specified downrange position. The reduced solutions corresponding to these problems are obtained by maximizing (with respect to $\mathrm{V}$ and $\eta$ ) at each energy level the quantities [(T-D)V]/f for the former and $[(T-D) V] /\left(\mathrm{fV}_{0}-\mathrm{f}_{0} \mathrm{~V}\right)$ for the latter, where $\mathrm{V}_{0}$ is the most fuel efficient cruising speed of the aircraft and $\mathrm{f}_{0}$ is the fuel consumption rate at this cruising flight condition ${ }^{7}$. It is interesting to note the very low levels of $\varepsilon$ in Fig. 6 (compare with Figs. 2 and 4), suggesting two time scale behavior for the entire envelope.

Finally, Figs. 7 and 8 show the results for a hypersonic vehicle model, used by NASA and called "the Langley Accelerator"13. The only optimization problem considered in this case was minimum fuel to a specified energy, the reduced solution corresponding to which is obtained by maximizing the quantity $[(\eta T-D) V] /(m f)$ at each energy level (mass is not constant in this case). A maximum dynamic pressure constraint of $2000 \mathrm{lbf}$ per square feet is imposed on the climb paths for this case. This particular vehicle 
model employs a multimode propulsion system, sized for acceleration to orbital velocity and consisting of turbojet, ramjet, scramjet, and rocket cycles. Note that Figs. 7 and 8 correspond to a nonoptimal switching between the different propulsion cycles. Specifically, we first assume allowable operating ranges for the different propulsion cycles (expressed as bounds on the Mach number), and then, at each energy level we pick the cycle that maximizes $[(\eta T-D) V] /(m f)$ and $(\eta T-D) / m$. The switching therefore from one cycle of propulsion to the other is abrupt, with no overlap. The actual points of cycle transitions are also shown in the figures. Note also that in Fig. $8 \varepsilon$ is plotted against the speeds at which the constant energy contours intersect the zero altitude axis. The reason for this is that $\mathrm{E}$ is negative in this case. The actual value of $\varepsilon$ is likely to be much lower if a practical method for cycle transition is employed. Note finally that as the energy levels get higher and higher we approach the boundary of the envelope and $\varepsilon$ goes to zero. This is basically a characteristic of all aircraft (see also Figs. 2, 4, and 6) suggesting that the transitions to the reduced solution can be treated as boundary layers more succesfully at high energy levels than at low ones. The physical explanation for this comes from the behavior of the difference between the thrust $T$ and the drag $D$. At low energy levels both the speed and altitude are low, implying that the thrust is high and the drag is low, so that T-D is high and can be used to change the energy during a transition. At high energy levels on the other hand either the speed or the altitude or both are high, implying that the difference T-D is low. Thus, transitions to the reduced solution at high energy levels can be expected to occur more or less by interchanging kinetic for potential energy (or vice versa), with the total energy staying nearly constant.

\section{Conclusions}

For both the conventional (subsonic-supersonic, flat Earth) and the transatmospheric (hypersonic, spherical Earth) flight regimes a systematic procedure was introduced to identify naturally a singular perturbation parameter $\varepsilon$ in the differential equations of motion. The procedure consists of using a set of arbitrary scaling constants to nondimensionalize all the variables of interest and then applying a set of conditions on these constants to put the resulting nondimensional equations of motion in the traditional singular perturbation format. Because the number of conditions is less than the number of constants, the 
scaling constants cannot be uniquely specified. Thus, the resulting expression for $\varepsilon$ is in general quite arbitrary. There is, however, a particular choice of the scaling constants for which the values of the resulting $\varepsilon$ can serve as a hint for a possible two-time-scale behavior of the aircraft in question. The primary result of the paper is the demonstration that for this "useful" choice of the scaling constants the resulting $\varepsilon$ is always equal to the maximum longitudinal loading factor of the vehicle, measured in units of g's. Two time scale behavior is suggested according to whether $\varepsilon$ is less than one or not. Based on this result it is straightforward to see why singular perturbation methods applied to aircraft performance optimization problems have worked so well in the past. The maximum longitudinal loading factors associated with the majority of conventional aircraft are either less than one because the aircraft lack very high thrusting capabilities, or because they are restricted to be so for other reasons (structural, comforting, etc.). A few notable exceptions do occur for some modern fighters. This directly suggests that most conventional aircraft can be expected to exhibit two-time-scale behavior for almost any energy climb that they are allowed to perform. This then appears to be the reason for the past success of so many singular perturbation treatments of aircraft energy climbs. The implication for transatmospheric vehicles is rather direct. If we consider such a vehicle as a passenger transport, then, in order to assure passenger comfort it is only natural to impose as a constraint a maximum longitudinal loading factor for the vehicle that is less than one. Our work suggests that such a constraint would imply two-time-scale behavior for any type of energy climb that such a vehicle would be allowed to perform. Therefore, singular perturbation formulations of such maneuvers still appear to be promising, even with all the added complexities that the flight regimes of such vehicles can entail.

\section{Acknowledgement}

This research was supported by NASA Langley Research Center under Grant No. NAG-1-922. The NASA technical monitor is Dr. Dan Moerder. 


\section{References}

1. Ardema, M. D., "Separation of Time Scales in Aircraft Trajectory Optimization," AIAA Journal of Guidance and Control, Vol. 8, No. 2, March-April 1985.

2. Shinar, J., "On Applications of Singular Perturbation Techniques in Nonlinear Optimal Control," Automatica, Vol. 19, No. 2, 1983.

3. Breakwell, J. V., "Optimal Flight-Path Angle Transitions in Minimum-Time Airplane Climbs," Journal of Aircraft, Vol. 14, August 1977.

4. Calise, A. J., "Optimal Thrust Control with Proportional Navigation Guidance," AIAA Journal of Guidance and Control, Vol. 3, No. 4, July-August 1980.

5. Kelley, H. J. and Edelbaum, T. N., "Energy Climbs, Energy Turns, and Asymptotic Expansions," Journal of Aircraft, Vol. 7, Jan-Feb 1970.

6. Ardema, M. D., "Solution of the Minimum Time-to-Climb Problem by Matched Asymptotic Expansions," AIAA Journal, Vol. 14, No. 7, July 1976.

7. Calise, A. J., "Extended Energy Management Methods for Flight Performance Optimization," AIAA Journal, Vol. 15, No. 3, March 1977.

8. Calise, A. J., "Singular Perturbation Techniques for On-Line Optimal Flight-Path Control," ALAA Journal of Guidance and Control, Vol. 4, No. 4, July-August 1981.

9. Corban, J. E., Calise, A. J., and Flandro, G. A., "Rapid Near-Optimal Trajectory Generation for Single-Stage-to-Orbit Airbreathing Vehicles," To appear in the AIAA Journal of Guidance, Control and Dynamics.

10. Calise, A. J., and Moerder, D. D., "Singular Perturbation Techniques for Real Time Aircraft Trajectory Optimization and Control," NASA CR-3597, August 1982.

11. Calise, A. J., and Pettengil, J. B., "A Comparison of Time-Optimal Intercept Trajectories for the F-8 and F-15 - Final Report," NASA Grant No. NCC 2-506, January 1990.

12. Barman, J. F., and Erzberger, H., "Fixed-Range Optimum Trajectories for Short-Haul Aircraft," Journal of Aircraft, Vol. 13, No. 10, October 1976. 
13. Corban, J. E., "Real-Time Guidance and Propulsion Control for Single-Stage-to-Orbit Airbreathing Vehicles," Ph.D. Dissertation, The Georgia Institute of Technology, December 1989. 


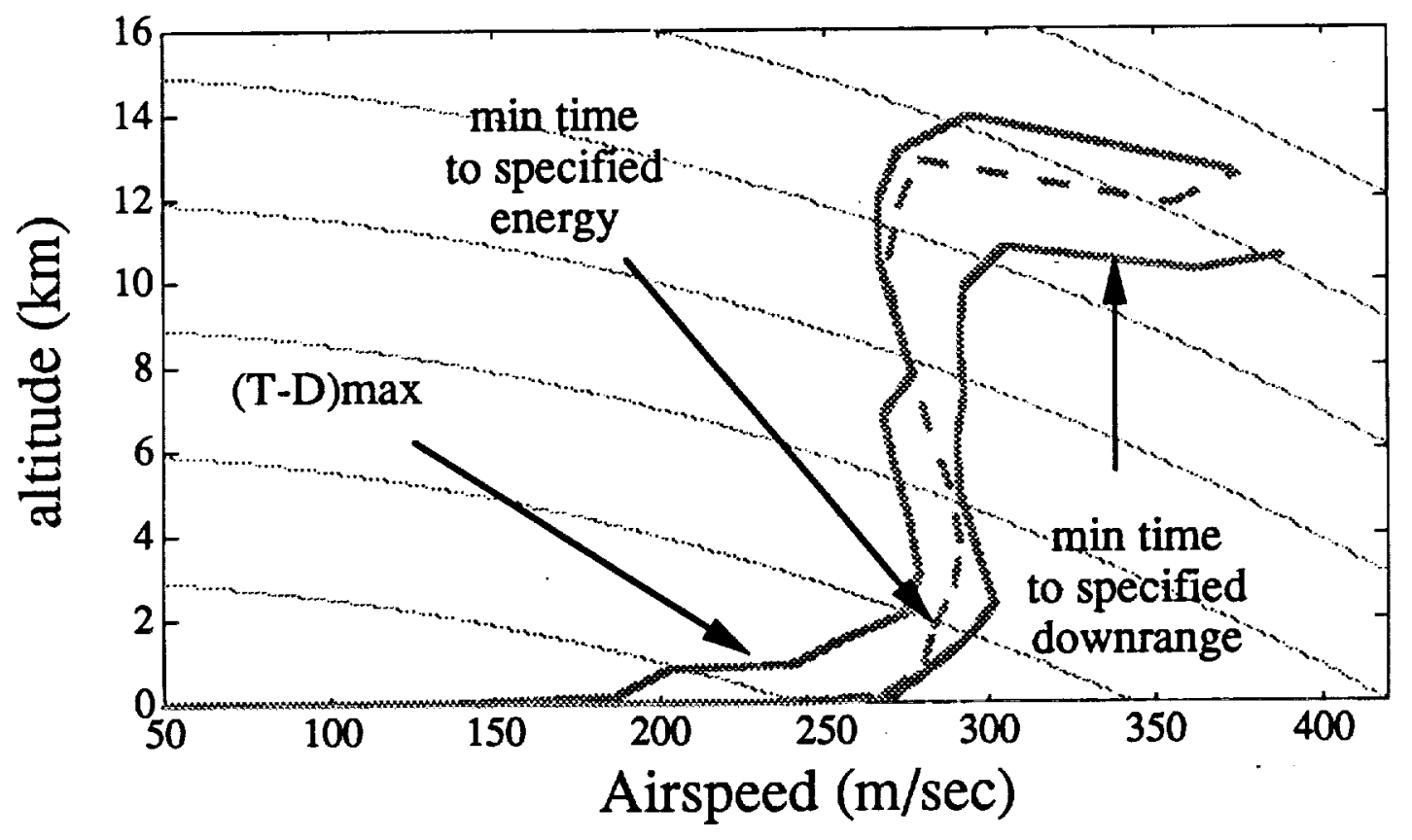

Fig. 1 Energy climb paths for an F-8 aircraft 


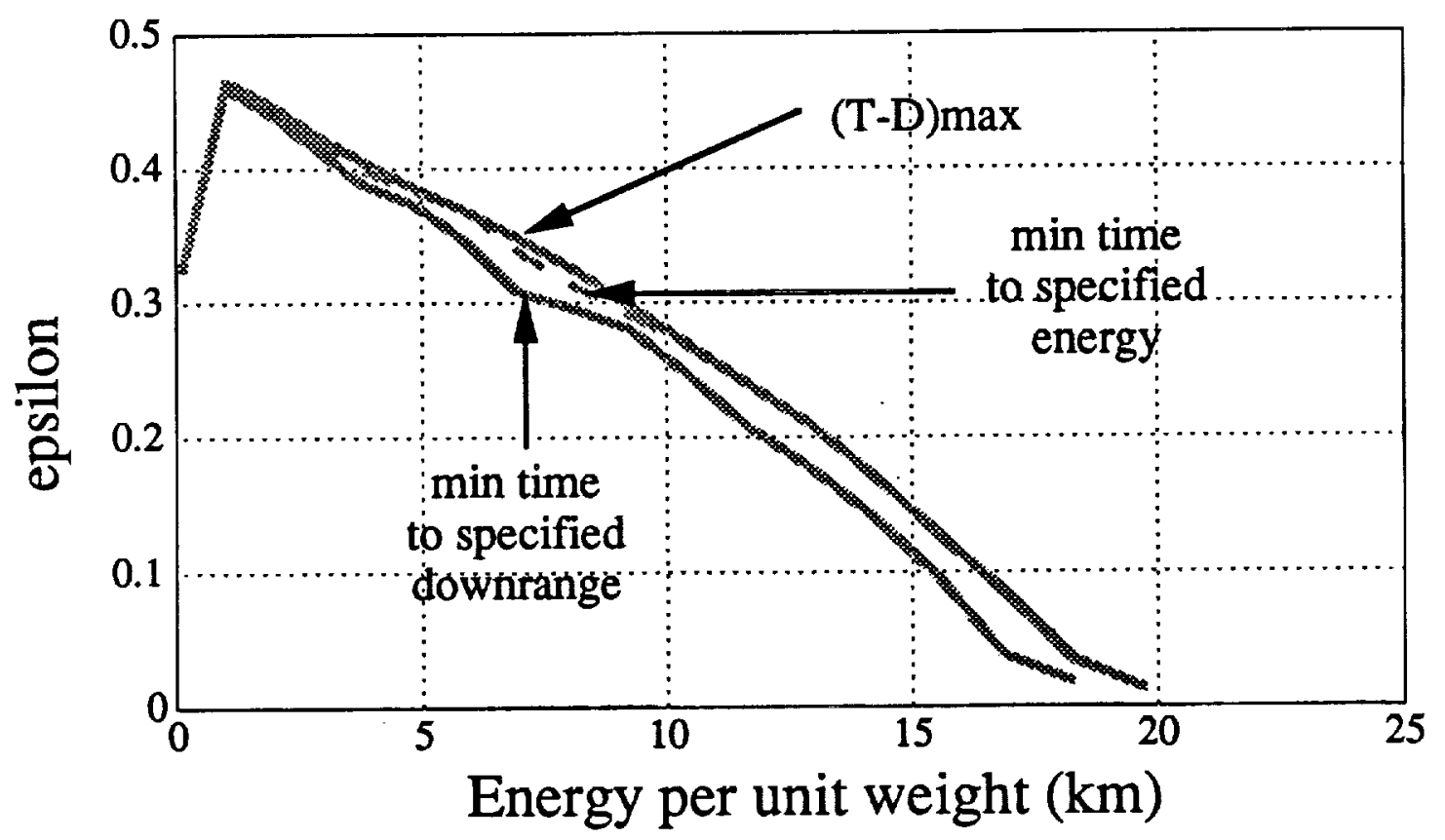

Fig. 2 Evaluation of $\varepsilon(E)$ for an F-8 aircraft 


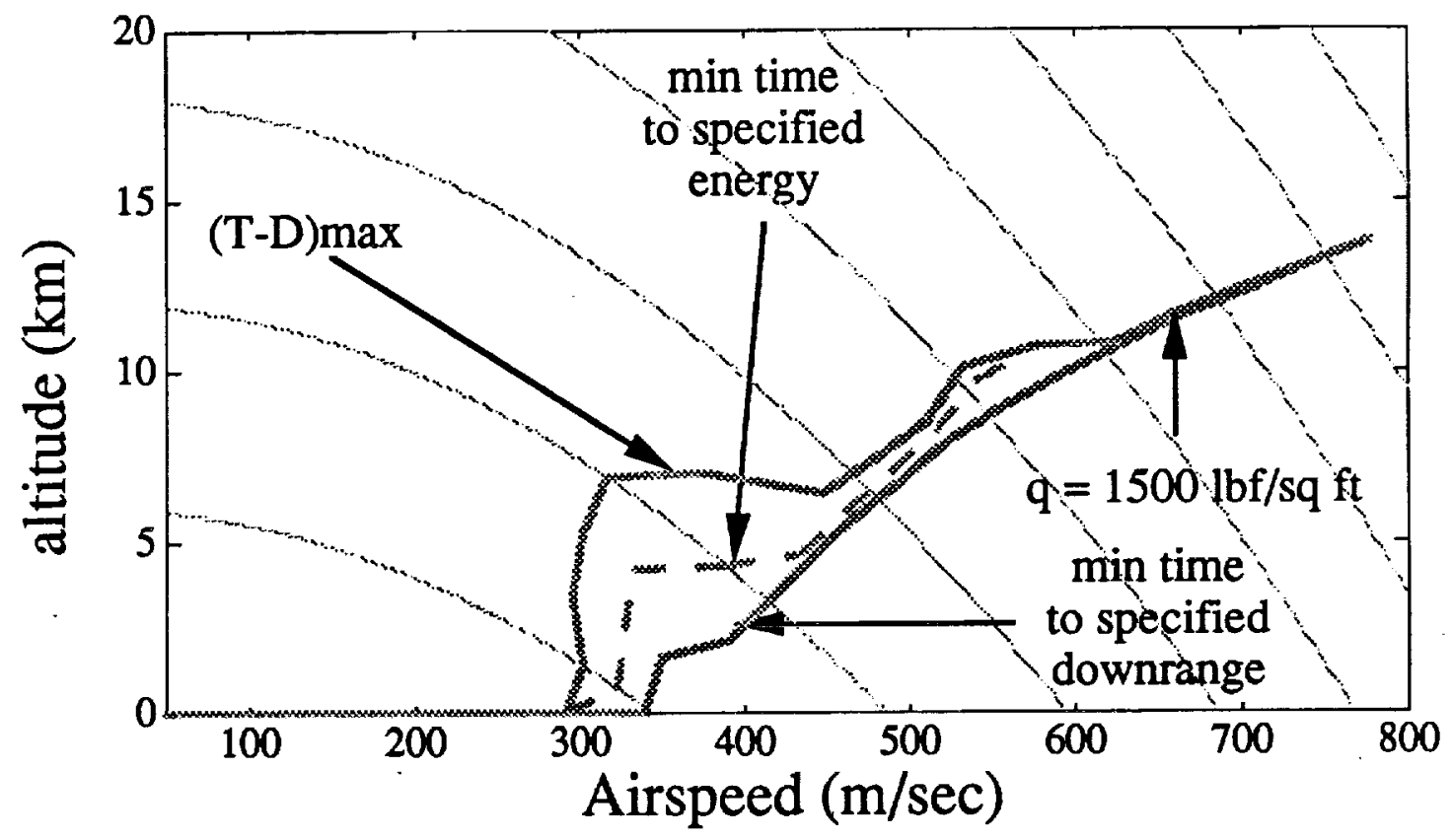

Fig. 3 Energy climb paths for an F-15 aircraft 


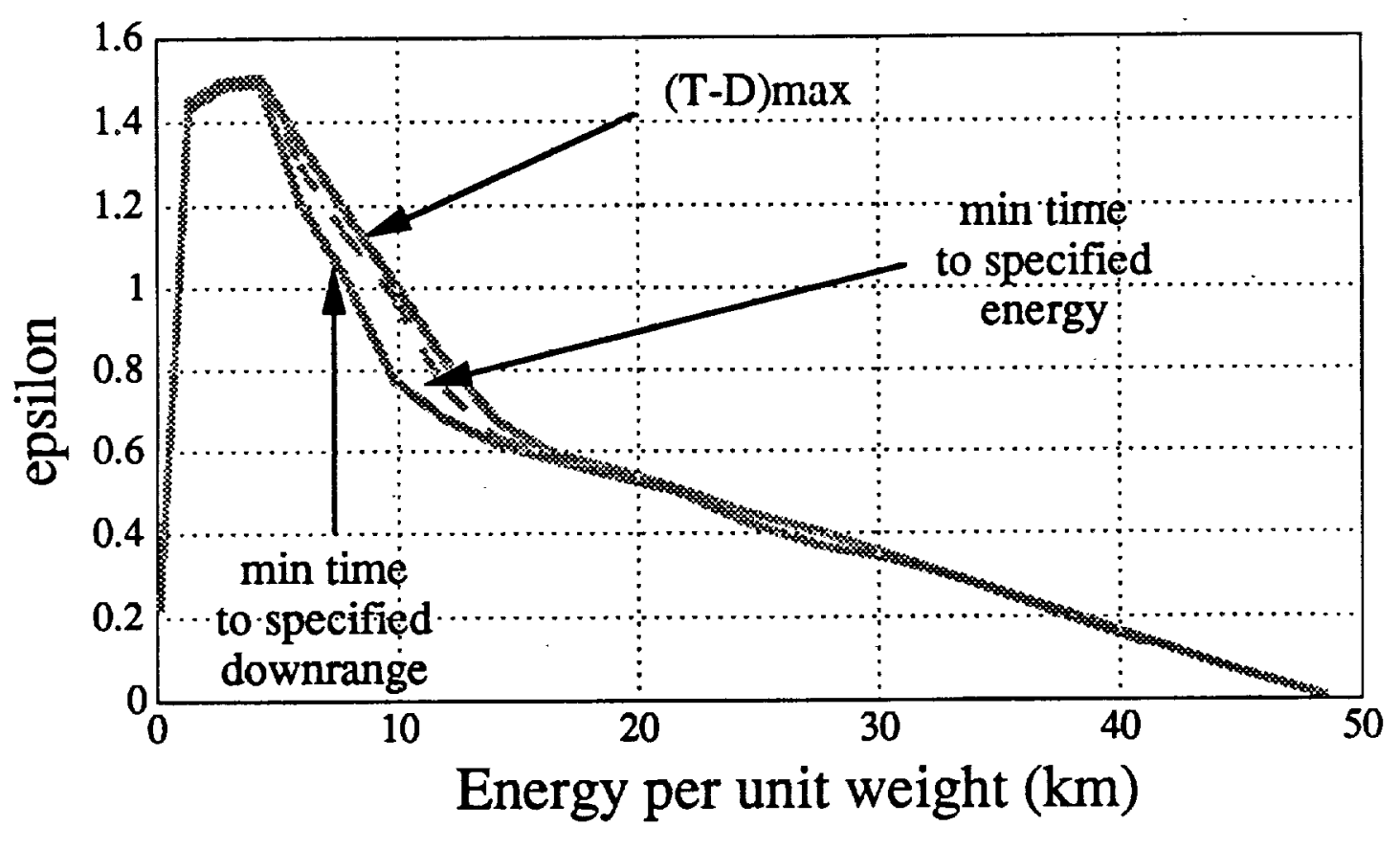

Fig. 4 Evaluation of $\varepsilon(E)$ for an F-15 aircraft 


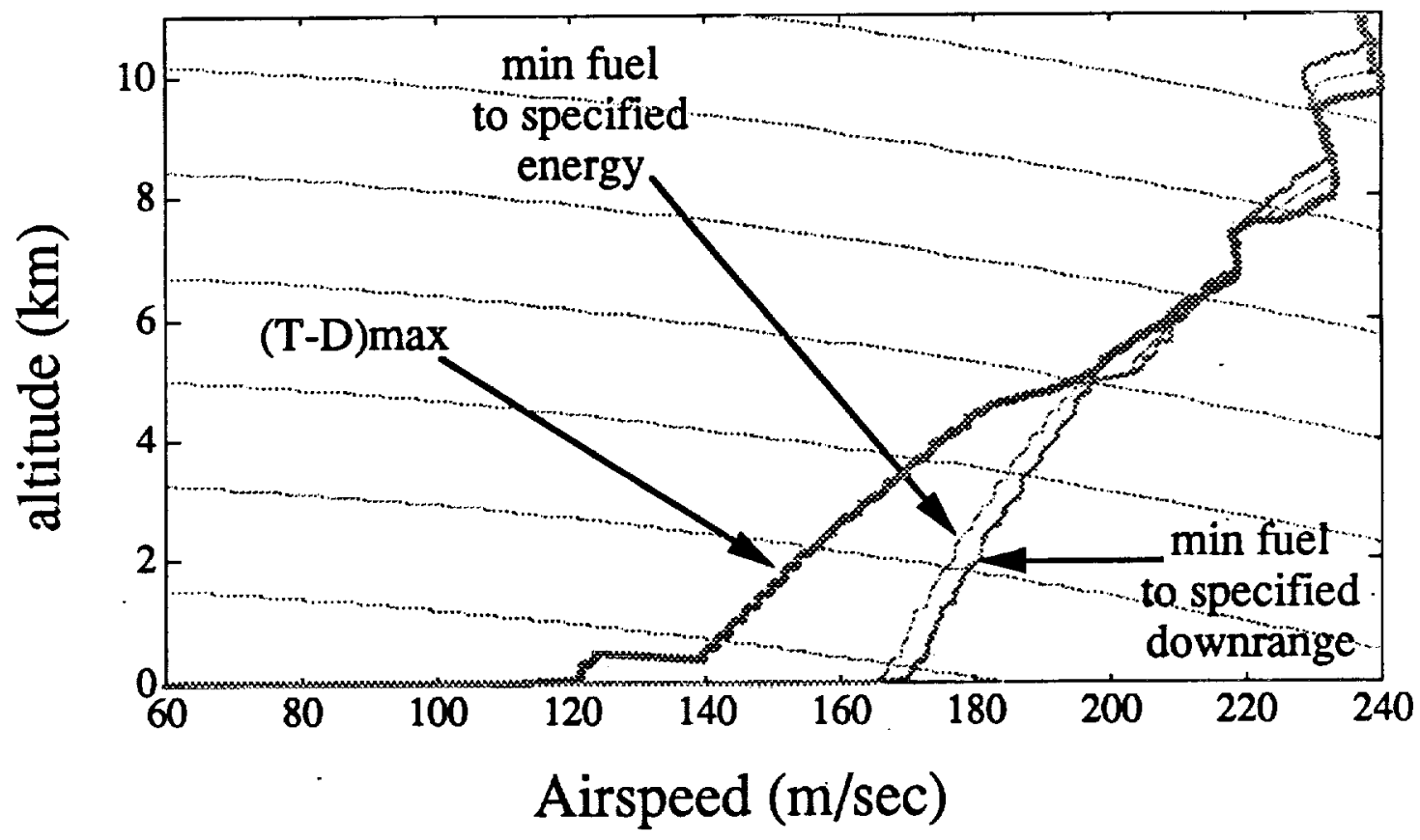

Fig. 5 Energy climb paths for a short-haul transport aircraft 


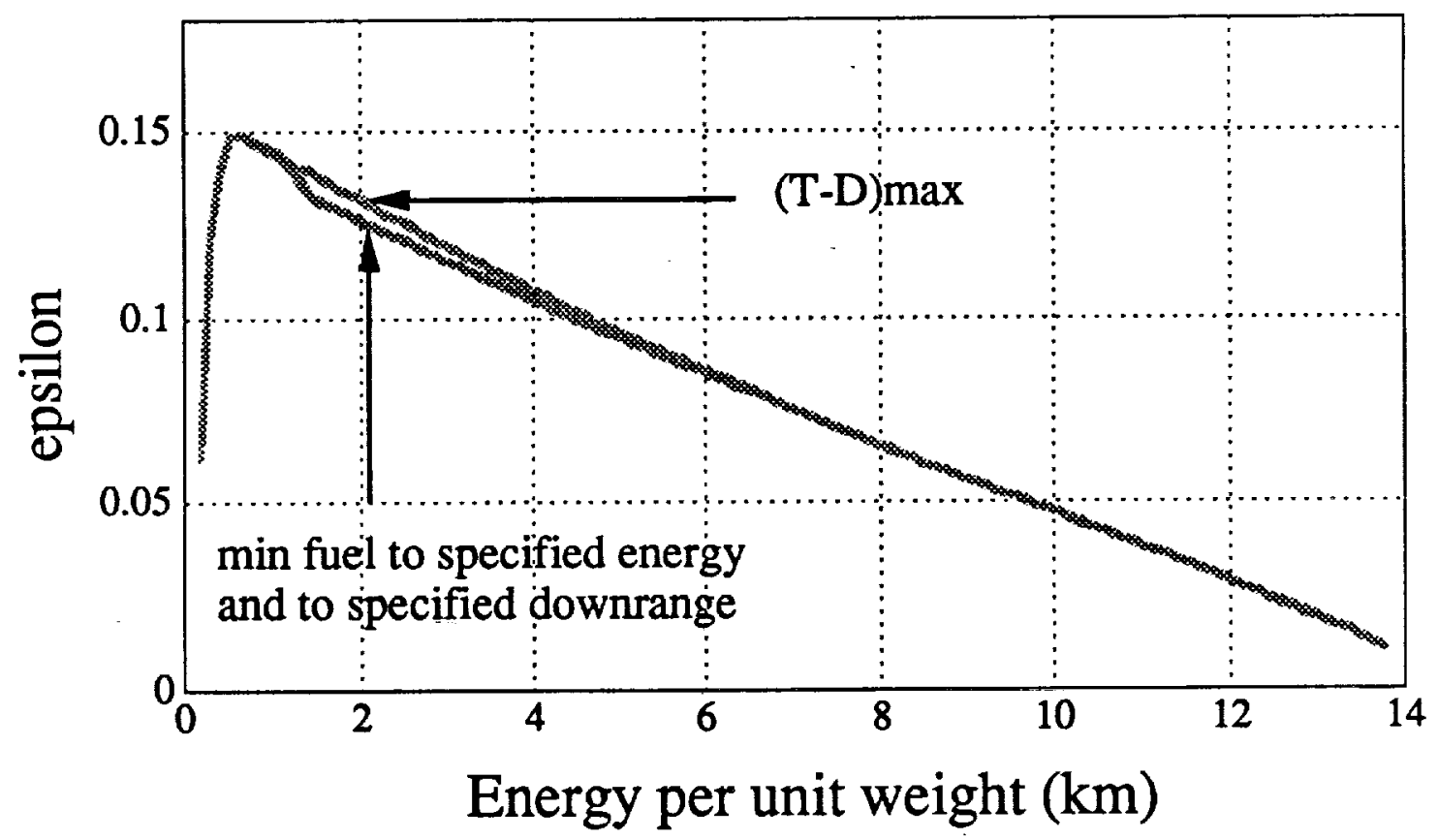

Fig. 6 Evaluation of $\varepsilon(E)$ for a short-haul transport aircraft 


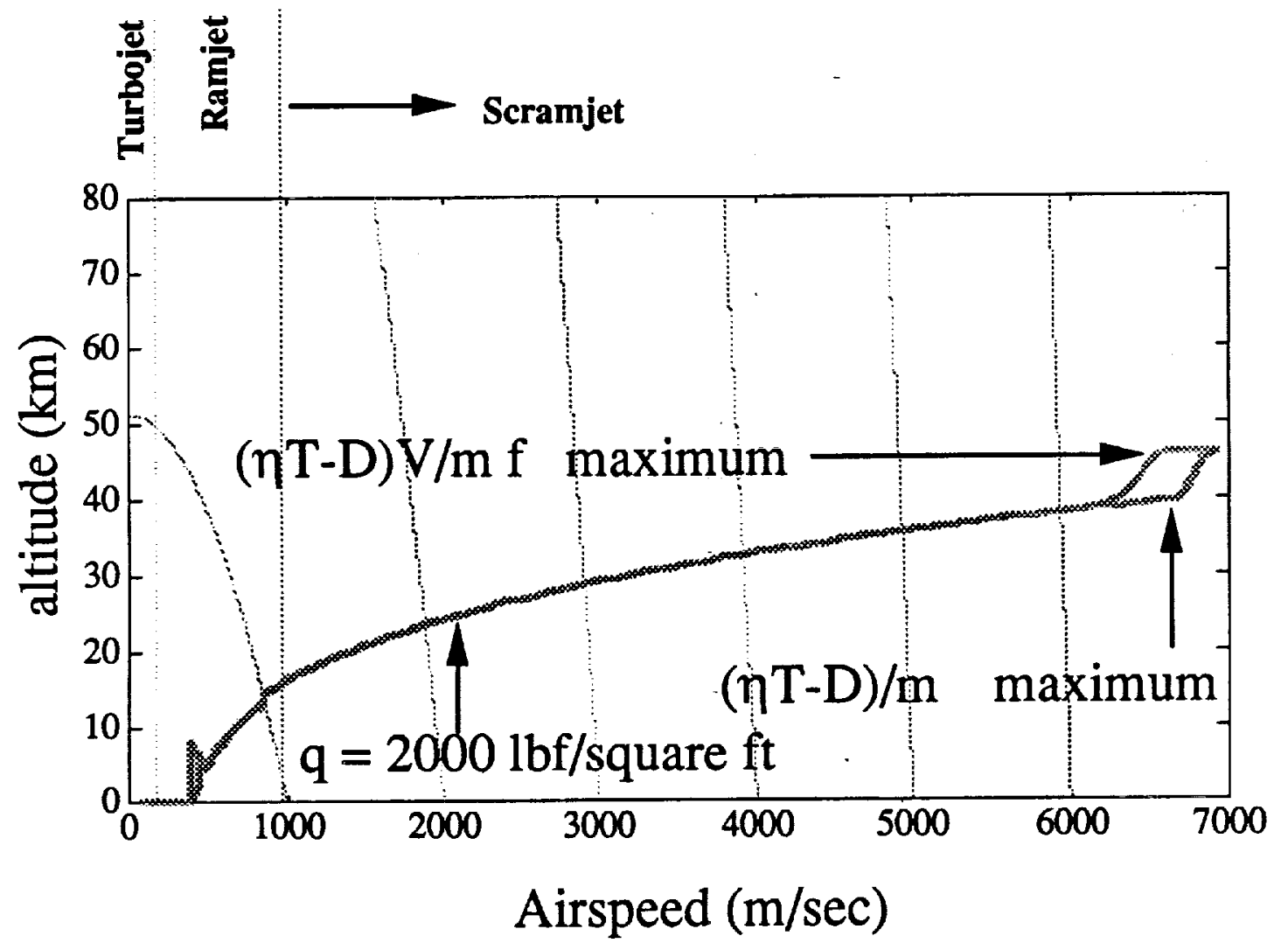

Fig. 7 Energy climb paths for a generic hypersonic vehicle 


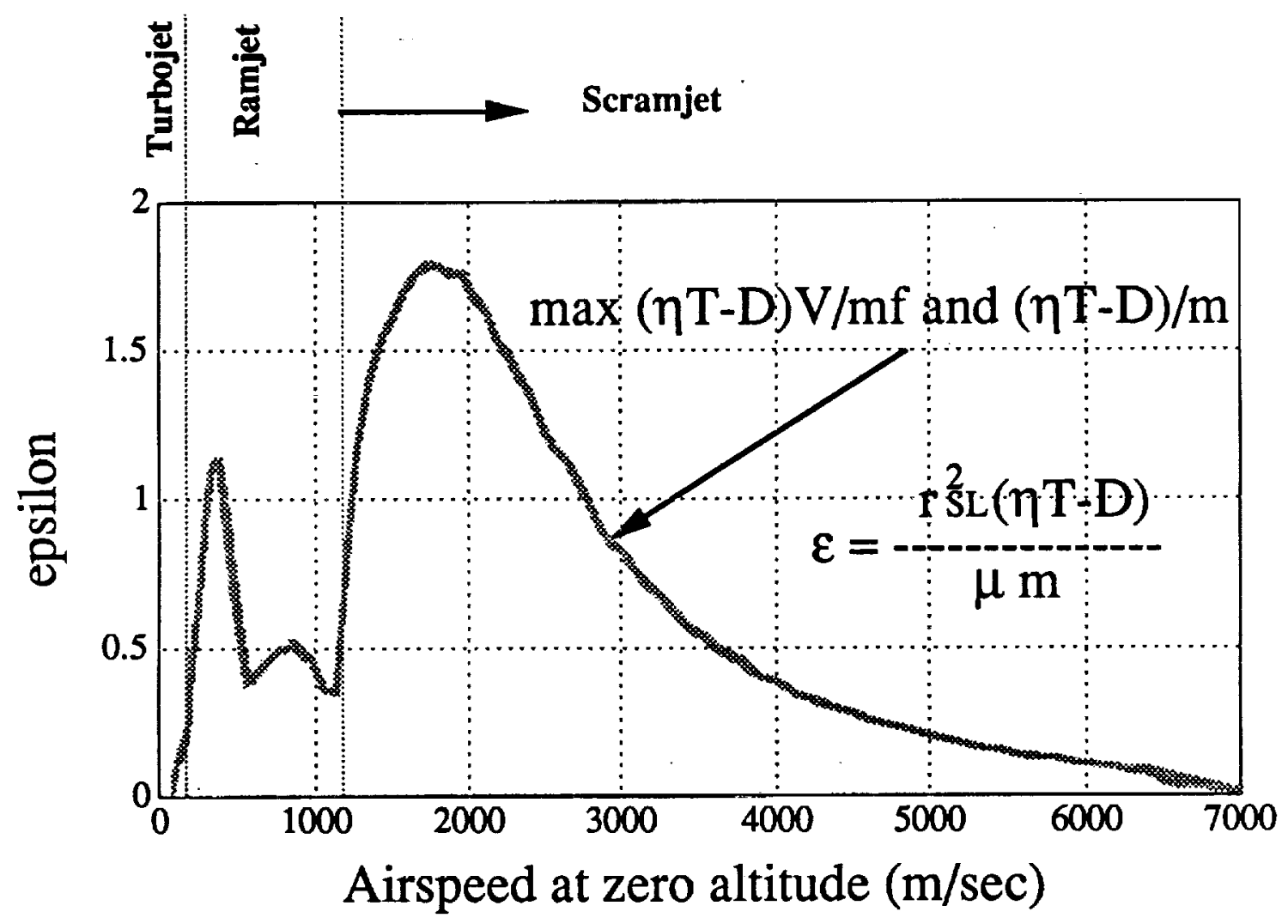

Fig. 8 Evaluation of $\varepsilon(E)$ for a generic hypersonic vehicle 